\title{
Ultrathin Single-Crystalline CdTe Nanosheets Realized via Van der Waals Epitaxy
}

\author{
Ruiqing Cheng, Yao Wen, Lei Yin, Fengmei Wang, Feng Wang, Kaili Liu, \\ Tofik Ahmed Shifa, Jie Li, Chao Jiang, Zhenxing Wang, * and Jun He*
}

Due to the novel physical properties, high flexibility, and strong compatibility with Si-based electronic techniques, 2D nonlayered structures have become one of the hottest topics. However, the realization of 2D structures from nonlayered crystals is still a critical challenge, which requires breaking the bulk crystal symmetry and guaranteeing the highly anisotropic crystal growth. CdTe owns a typical wurtzite crystal structure, which hinders the 2D anisotropic growth of hexagonal-symmetry CdTe. Here, for the first time, the 2D anisotropic growth of ultrathin nonlayered $\mathrm{CdTe}$ as thin as $4.8 \mathrm{~nm}$ via an effective van der Waals epitaxy method is demonstrated. The anisotropic ratio exceeds $10^{3}$. Highly crystalline nanosheets with uniform thickness and large lateral dimensions are obtained. The in situ fabricated ultrathin 2D CdTe photodetector shows ultralow dark current $(\approx 100 \mathrm{fA})$, as well as high detectivity, stable photoswitching, and fast photoresponse speed $\left(\tau_{\text {rising }}=18.4 \mathrm{~ms}, \tau_{\text {decay }}=14.7 \mathrm{~ms}\right)$. Besides, benefitting from its 2D planar geometry, CdTe nanosheet exhibits high compatibility with flexible substrates and traditional microfabrication techniques, indicating its significant potential in the applications of flexible electronic and optoelectronic devices.

the relatively weak van der Waals interaction between adjacent layers, 2D ultrathin layered materials can be easily obtained via mechanical exfoliation or chemical vapor deposition. However, there are also many nonlayered materials with unique properties that are different from layered materials. For example, many nonlayered crystals, such as III-V group semiconductors (GaN), II-VI group materials ( $\mathrm{ZnO})$, and others, own superior optoelectronics properties and have been widely used in electronic and optoelectronic devices. ${ }^{[15]}$ Because charge carriers, phonon, and photon transports are strongly confined into 2D plane, 2D structures may bring some new physical phenomena, such as 2D electron gas and quantum hall effects. ${ }^{[16,17]}$ CdTe is acknowledged for exhibiting excellent optoelectronic performance emanated from its near-optimum direct bandgap and high absorption coefficient, which makes it an ideal candidate

Ultrathin 2D materials, including graphene, ${ }^{[1,2]} \mathrm{MoS}_{2},{ }^{[3,4]}$ $\mathrm{WS}_{2},{ }^{\left[{ }^{[5]}\right.}$ black phosphorus, ${ }^{[6]}$ etc., have triggered worldwide interests due to their fascinating thickness-dependent optical and electrical properties and indicated great potential in various applications, such as field-effect transistors (FETs), ${ }^{[7]}$ memories, ${ }^{[8,9]}$ sensors, ${ }^{[10]}$ photodetectors, ${ }^{[11,12]}$ photovoltaic devices, ${ }^{[13]}$ and so on. Additionally, their 2D planar structures not only own high compatibility with flexible substrates and traditional silicon microfabrication techniques, but also provide a possibility of preparing novel artificial materials via stacking different ultrathin layers. ${ }^{[14]}$ At the present time, the study of 2D nanostructures is mainly limited to the naturally layered materials. Due to the strong in-plane covalent bonding and for photovoltaic and photodetection applications. For example, monocrystalline CdTe solar cells with open-circuit voltage over $1 \mathrm{~V}$ and efficiency of $17 \%$ were obtained. ${ }^{[18]}$ Moreover, 2D electron gas and consequent quantum oscillations have been found at $\mathrm{PbTe} / \mathrm{CdTe},{ }^{[19]} \mathrm{InSb} / \mathrm{CdTe},{ }^{[20]}$ and $\mathrm{HgTe} / \mathrm{CdTe}$ interfaces, ${ }^{[21]}$ which is potential for use in high electron mobility transistors (HEMTs). Therefore, the controlled synthesis of 2D nonlayered CdTe is significantly important and highly desired. In comparison with van der Waals structure of 2D layered materials, nonlayered CdTe owns typical wurtzite structure (Figure 1a) formed via covalent bonding. When the thickness goes down to several nanometers, it results in lots of unsaturated dangling bonds on the surface, which makes the $2 \mathrm{D}$ anisotropic growth quite
R. Cheng, L. Yin, F. M. Wang, F. Wang, K. Liu, T. A. Shifa,

J. Li, Prof. Z. Wang, Prof. J. He

CAS Center for Excellence in Nanoscience

CAS Key Laboratory of Nanosystem and Hierarchical Fabrication

National Center for Nanoscience and Technology

Beijing 100190, P. R. China

E-mail:wangzx@nanoctr.cn; hej@nanoctr.cn

R. Cheng, Y. Wen, L. Yin, F. M. Wang, F. Wang, K. Liu,

T. A. Shifa, J. Li, Prof. J. He

University of Chinese Academy of Sciences

Beijing 100049, P. R. China
R. Cheng, K. Liu

Sino-Danish Center for Education and Research

Beijing 100190, P. R. China

Y. Wen, Prof. C. Jiang

CAS Center for Excellence in Nanoscience

CAS Key Laboratory for Standardization and Measurement

for Nanotechnology

National Center for Nanoscience and Technology

Beijing 100190, P. R. China

The ORCID identification number(s) for the author(s) of this article can be found under https://doi.org/10.1002/adma.201703122.

DOI: 10.1002/adma.201703122 

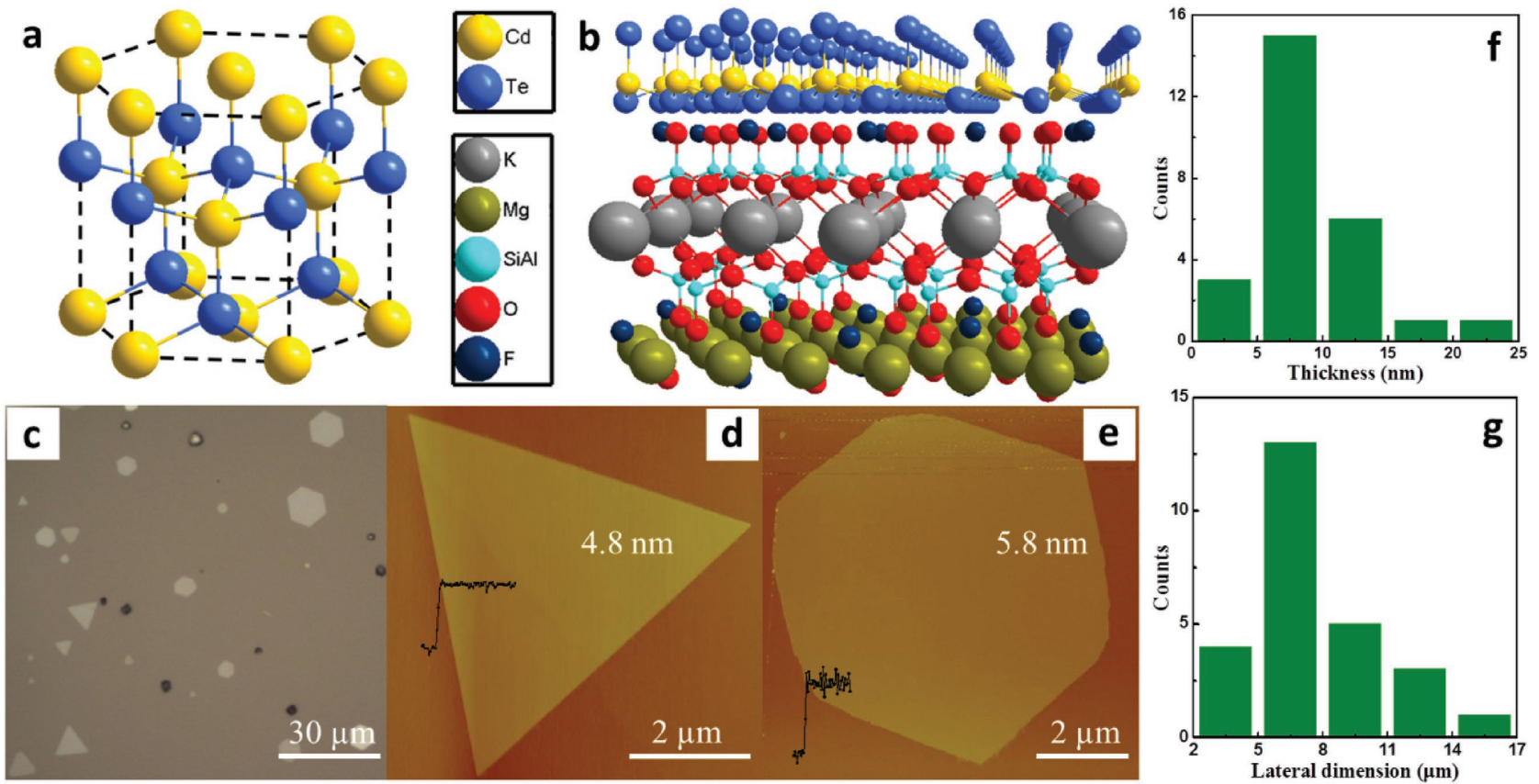

Figure 1. Morphologies of 2D CdTe nanosheets. Schematic illustrations of a) CdTe with wurtzite structure and b) epitaxial 2D CdTe nanosheet (top) grown on mica substrate (bottom). c) OM image of CdTe nanosheets on mica substrate. d,e) AFM images and height profiles of two typical CdTe nanosheets. The thickness was determined to be 4.8 and $5.8 \mathrm{~nm}$, respectively. The histogram statistics of the f) thickness and the g) lateral dimension of 2D CdTe nanosheets obtained from a substrate area of $\approx 0.5 \mathrm{~mm} \times 0.5 \mathrm{~mm}$.

difficult. So it is still a critical challenge to achieve ultrathin nanosheets from nonlayered crystals.

Numerous methods to grow 2D structures from nonlayered materials have been reported. They have been employed to grow metals, oxides, and semiconductors ${ }^{[22]}$ For instance, a solution-based templated method, so called adaptive ionic layer epitaxy (AILE), has been developed to synthesize $\approx 1-2 \mathrm{~nm}$ thick, oleylsulfate-adsorbed $\mathrm{ZnO}$ nanosheets. ${ }^{[23]}$ However, this method requires the introduction of surfactants or polymers that are detrimental for obtaining clean and well-defined interfaces, making them inoperable for perfectly stacking 2D heterostructures. Another example, Balushi et al. demonstrated the synthesis of atomically thin 2D GaN on the $\mathrm{SiC}$ substrates through a migration-enhanced encapsulated growth (MEEG) technique. ${ }^{[24]}$ But, the small lateral size (less than $500 \mathrm{~nm}$ ) and strong interaction with $\mathrm{SiC}$ substrates bring myriad fabrication challenges for further component processing.

Aiming at weakening the interaction between overlayers and surfactant templates or substrates, van der Waals epitaxy (vdWE) is considered as an effective method for the controllable growth of ultrathin 2D nanosheets from nonlayered crystals, which makes it possible that these nonlayered materials compatible with flexible substrates and silicon technology. In contrast to traditional epitaxial growth, requiring strict lattice matching, vdWE is based on the weak van der Waals interaction between the epitaxial 2D materials and substrates, which enable the growth of defect-free products. ${ }^{[25,26]}$ Moreover, this weak interaction between overlayers and substrates allows the easy release of products for transferring onto other substrates or ultrathin 2D materials. VdWE has succeeded in the growth of 2D layered materials (such as GaSe, ${ }^{[27]} \mathrm{MoS}_{2},{ }^{[28]} \mathrm{In}_{2} \mathrm{Se}_{3},{ }^{[29]}$
$\left.\mathrm{Bi}_{2} \mathrm{O}_{2} \mathrm{Se}\right)^{[30]}$ and 2D nonlayered materials (such as $\mathrm{Te}^{[31]}$ $\left.\mathrm{Pb}_{1-x} \mathrm{Sn}_{x} \mathrm{Se},{ }^{[32]} \mathrm{CdSe},{ }^{[33]} \mathrm{PbS}\right) \cdot{ }^{[34]}$ However, the realization of nanometer-thick single-crystalline $2 \mathrm{D}$ nonlayered materials via vdWE has remained a great challenge.

Here, for the first time, we demonstrate the synthesis of large-size ultrathin CdTe nanosheets with wurtzite crystal structure as thin as $\approx 4.8 \mathrm{~nm}$ via vdWE method. The anisotropic ratio exceeds $10^{3}$. The in situ fabricated two-terminal ultrathin CdTe photodetector shows ultralow dark current $(\approx 100 \mathrm{fA})$, as well as high detectivity, stable photoswitching, and ultrafast photoresponse speed $\left(\tau_{\text {rising }}=18.4 \mathrm{~ms}, \tau_{\text {decay }}=14.7 \mathrm{~ms}\right)$, indicating its remarkable potential in optoelectronics devices. Besides, benefitting from its 2D planar geometry, the obtained CdTe nanosheets exhibit high compatibility with flexible substrates and traditional microfabrication techniques, suggestive of its tangible application in optoelectronic devices.

Figure $1 \mathrm{~b}$ exhibits the schematic illustration of vdWE growth of nonlayered CdTe nanosheets on mica substrate. CdTe nanosheets are prepared by vapor transport method. The mixture of high-purity CdTe and Te powders was used as a precursor. The Te powder plays a crucial role in the large-size epitaxial growth of CdTe nanosheets. Only few small-sized 2D nanostructures are found without such Te powder keeping the same of other experimental conditions. This phenomenon can be interpreted by our previous work wherein we demonstrated that Te atoms tend to gather into nanoisland, which is in favor of the growth of 2D structure. ${ }^{[31]}$ Layered fluorophlogopite mica $\left[\mathrm{KMg}_{3}\left(\mathrm{AlSi}_{3} \mathrm{O}_{10}\right) \mathrm{F}_{2}\right]$ sheets with pseudohexagonal $\mathrm{Z}_{2} \mathrm{O}_{5}$ layers $(\mathrm{Z}=\mathrm{Al}, \mathrm{Si})$ were used as growth substrates. Due to the absence of surface dangling bonds, the exposed (001) face of mica exhibits chemical inertness and atomically smooth surface, as 
shown in Figure 1b (bottom). Consequently, small migration energy barrier of adatoms along the mica surface is achieved, facilitating the high-speed migration of adatoms and fast lateral growth of nonlayered materials. This can be verified by the relation of migration coefficient $\left(D_{\mathrm{m}}\right)$ and migration barrier energy $\left(E_{\mathrm{m}}\right)$ of adatoms ${ }^{[32]}$

$$
D_{\mathrm{m}} \propto e^{\left(\frac{-E_{\mathrm{m}}}{k T}\right)}
$$

Here, $k$ is the Boltzmann constant and $T$ is the temperature of substrate. Since the growth rate along the lateral direction is much faster than vertical direction, we can achieve the 2D anisotropic growth of CdTe. More experimental details can be found in the Experimental Section. Figure 1c shows the optical microscope $(\mathrm{OM})$ image of the as-obtained CdTe nanosheets on the transparent mica substrate. It is apparent from the OM image that CdTe nanosheets appear as regular triangle and hexagon geometry, which are in agreement with the intrinsic wurtzite structure of CdTe. The morphologies and thickness of nanosheets are identified by atomic force microscope (AFM). A typical regular triangular nanosheet with thickness of $4.8 \mathrm{~nm}$ and a subhexagonal nanosheet with thickness of $5.8 \mathrm{~nm}$ are presented in Figure 1d,e, respectively. As a result of the weak van der Waals interaction between overlayers and mica substrates, CdTe nanosheet exhibits a flat surface with a uniform thickness. The corresponding anisotropic ratios, which are defined as the ratio of lateral size to vertical thickness, are estimated to be $\approx 1290$ and $\approx 1350$, respectively. These high ratios indicate the highly anisotropic 2D growth of CdTe. Figure 1f,g exhibits the histogram statistics of the thickness distribution and the lateral dimension distribution of 26 CdTe nanosheets obtained from a substrate area of $\approx 0.5 \mathrm{~mm} \times 0.5 \mathrm{~mm}$. The original AFM images are given in Figure S1 (Supporting Information). The thickness and lateral dimension are mainly distributed in $\approx 5-10 \mathrm{~nm}$ and $\approx 5-8 \mu \mathrm{m}$, respectively. Compared with other previous $2 \mathrm{D}$ nonlayered materials obtained by vdWE, the single-crystalline CdTe nanosheets we obtained for the first time approach sub-10 nm thickness or lower. The observed large lateral dimension and flat surface give rise to the realization of combining CdTe nanosheet with other ultrathin 2D materials, which may provide promising applications in high-performance photovoltaic devices, especially constructing layered 2D/nonlayered 2D heterostructures.

High-resolution transmission electron microscopy (HRTEM) is applied to investigate the detailed information about the structure of $\mathrm{CdTe}$ nanosheets. As shown in Figure 2a, the HRTEM image shows a perfect hexagonal lattice fringes with a periodic space of $3.7 \AA$, matching well with its (002) plane (PDF No. 19-0193). The corresponding selected area electron diffraction (SAED) pattern (the inset of Figure 2a) exhibits high-quality single-crystalline hexagonal phase, indicating the wurtzite structure of CdTe nanosheets. Its chemical composition is characterized by elemental quantitative analysis of TEM energy-dispersive X-ray spectroscopy (TEM-EDX), showing that the atomic percentage ratio of $\mathrm{Cd}$ to $\mathrm{Te}$ is $1.075: 1$, which is in good agreement with the stoichiometric ratio of CdTe. From the TEM-EDX elemental mapping (Figure 2b), we found that $\mathrm{Cd}$


Figure 2. Characterizations of 2D CdTe nanosheets. a) HRTEM image of CdTe nanosheets with corresponding SAED as the inset. Lattice constant of (002) plane is $0.37 \mathrm{~nm}$. b) TEM-EDX mapping of tellurium and cadmium in CdTe nanosheet. c,d) XPS of CdTe nanosheets. e) Raman spectra of CdTe nanosheet excited by $532 \mathrm{~nm}$ laser. f) PL spectra of CdTe nanosheet excited by $514 \mathrm{~nm}$ laser. 
and Te are uniformly distributed in the entire nanosheet. Considering that the triangular crystals could also be (111)-oriented zinc blende, the structure of 2D CdTe triangular nanosheet was also examined. Corresponding TEM image and SAED pattern are given in Figure S2 (Supporting Information) and also exhibit wurtzite structure, indicating that triangular and hexagonal nanosheets own the same crystal structure. The chemical states and composition were further confirmed by X-ray photoelectron spectroscopy (XPS) analysis. In Figure 2c, the two peaks at 585.1 and $574.7 \mathrm{eV}$ clearly indicate the existence of Te $3 \mathrm{~d}_{3 / 2}$ and Te $3 \mathrm{~d}_{5 / 2}$ from CdTe. ${ }^{[35]}$ While the other two peaks at higher binding energies of 588.6 and $578.1 \mathrm{eV}$ are assigned to the tellurium oxide that is formed due to surface oxidation. ${ }^{[35-37]}$ As shown in Figure 2d, two peaks stemmed from $\mathrm{Cd} 3 \mathrm{~d}_{3 / 2}$ and $\mathrm{Cd} 3 \mathrm{~d}_{5 / 2}$ states in CdTe are observed at binding energies of 413.1 and $406.3 \mathrm{eV}$, respectively. ${ }^{[35]}$ Similar to tellurium oxide, the other two peaks located at higher binding energies of 414.4 and $407.6 \mathrm{eV}$ are likely originated from cadmium oxide. ${ }^{[38]}$ This strong splitting can be attributed to the abundant unsaturated dangling bonds on the surface of ultrathin nanosheets, which are easily oxidized. Notably, the atomic ratio of Cd to Te obtained from XPS is found to be about 1.10:1, matching well with the result of TEM-EDX. Raman spectra of a CdTe nanosheet excited by $532 \mathrm{~nm}$ laser are given in Figure 2e. For the sake of unambiguously identifying the signal meant for CdTe, Raman spectra of pure mica sheet were obtained and the result is depicted along with the sample under investigation. As can be seen, three prominent Raman peaks are observed at 128,145 , and $168 \mathrm{~cm}^{-1}$, which correspond to the $A_{1}$ and $E$ modes of Te, and the longitudinal optical (LO) phonon mode of $\mathrm{CdTe}$, respectively. It is noted that Te-Raman modes are usually detected in CdTe samples, which may originate from the presence of little Te precipitates. ${ }^{[35,39]}$ This peak position agrees well with the previously reported one but with a little blue shift due to the increase of confinement in the 2D ultrathin structure. ${ }^{[35,39-41]}$ And its second-order (2LO) mode around $340 \mathrm{~cm}^{-1}$ is always associated with the peak of mica substrates, which makes it difficult to be distinctly identified. The photoluminescence (PL) spectra of CdTe nanosheets, excited by $514 \mathrm{~nm}$, are also used to identify its band structure. The PL spectra of $\approx 6 \mathrm{~nm}$ thick CdTe nanosheet are provided in Figure S3a (Supporting Information). By the deconvolution of the broad peak, it generates two Gaussian components centered at 725 and $795 \mathrm{~nm}$. The peak at $725 \mathrm{~nm}$ in our work should be attributed to the mica substrate, which can be verified from the PL spectra of a pure mica sheet (Figure S3b, Supporting Information). While the PL peak at $795 \mathrm{~nm}$ is stemmed from CdTe. The strong PL signals of mica substrate suppress the typical peak of ultrathin CdTe nanosheets, and hence it is difficult to elucidate the particularity of CdTe grown on mica sheet. In order to extract the required information from $\mathrm{PL}$, the CdTe nanosheets were transferred onto a $300 \mathrm{~nm} \mathrm{SiO} / 2 / \mathrm{Si}$ substrate. Accordingly, Figure $2 \mathrm{f}$ exhibits the PL spectra of a transferred CdTe nanosheet with thickness around $34 \mathrm{~nm}$. A single peak located at $801 \mathrm{~nm}$ $(1.55 \mathrm{eV})$ is observed, confirming its intrinsic direct bandgap nature. This value agrees well with the previously reported value of $1.50 \mathrm{eV}$ but with a little blue shift, which is probably due to the ultrathin structure of CdTe nanosheets. ${ }^{[18]}$ Besides, the PL spectra of CdTe powders are also characterized for comparison. The corresponding results shown in Figure S3c (Supporting Information) reveal a PL peak at $822 \mathrm{~nm}(1.51 \mathrm{eV})$. Considering this remarkable PL behavior of CdTe nanosheets, superior optoelectronic properties are expected.

Figure 3a shows the OM image of the in situ fabricated twoterminal ultrathin CdTe photodetector on the mica substrate. The corresponding AFM image and height profiles are given in the inset. It is obvious from the figure that the thickness of $\mathrm{CdTe}$ nanosheet is identified to be $14.3 \mathrm{~nm}$. Figure $3 \mathrm{~b}$ shows the current versus voltage bias $\left(I_{\mathrm{sd}}-V_{\mathrm{sd}}\right)$ curves of $2 \mathrm{D} \mathrm{CdTe}$ photodetector in the dark and in the presence of $473 \mathrm{~nm}$ lasers with different laser intensities $(P)$ at room temperature. The linear $I_{\mathrm{sd}}-V_{\mathrm{sd}}$ characteristics indicate the near ohmic behavior of our device. This ultrathin 2D CdTe photodetector exhibits an ultralow dark current $\left(I_{\text {dark }}: \approx 100 \mathrm{fA}\right)$. To further demonstrate the photoresponse with the laser on and off, a voltage bias of $1 \mathrm{~V}$ and different laser power intensities are applied. As shown in Figure 3c, with the on/off switching of incident laser, a highly stable and reversible photoswitching behavior between two states is demonstrated. However, signals for the current could not be detected timely with our instruments due to the ultralow dark current values. Thus, an oscilloscope method was adopted to obtain its real response time as previously reported. ${ }^{[42]}$ The corresponding result is given in the inset of Figure $3 \mathrm{~d}$, whose main part shows the plot of a separated response and reset cycle. The response time $\left(\tau_{\text {rising }}\right)$ and recovery time $\left(\tau_{\text {decay }}\right)$ are identified to be 18.4 and $14.7 \mathrm{~ms}$. This fast photoswitching feature emanated from the high crystalline quality of CdTe nanosheets we obtained. Generally, crystals with defect states suffer from the issue that the photogenerated carriers will be filled and released from the trap states during laser on and off. These phenomena, thus, prolong the response and recovery time. The investigation of optoelectronic properties in the extreme environment is particularly important for assessing the applicability of our material for intended purpose. To this end, we made such investigation and the result is depicted in Figure 3e,f. As can be seen, when the temperature decreases to $80 \mathrm{~K}, 2 \mathrm{D}$ CdTe photodetector exhibits lower dark current, which is below the detection limit of our instruments (less than $10 \mathrm{fA}$ ). It is worth noting that voltage bias of 1 and $2 \mathrm{~V}$ were used for room temperature and $80 \mathrm{~K}$ conditions, respectively. The photocurrent $\left(I_{\mathrm{ph}}\right)$, which is defined as the difference between drain currents with and without laser $\left(I_{\mathrm{ph}}=I_{\text {laser }}-I_{\mathrm{dark}}\right)$, was extracted and shown in Figure S4 (Supporting Information). The quasi-linear relation between $I_{\mathrm{ph}}$ and laser intensities indicates a typical photon-dependent behavior of CdTe photodetector; that is, more carriers are generated with more photons. To quantify the photoresponse property of $2 \mathrm{D}$ $\mathrm{CdTe}$ photodetector, corresponding important parameters, such as photoswitching ratio $\left(I_{\text {laser }} / I_{\text {dark }}\right)$, responsivity $(R)$, and specific detectivity $\left(D^{*}\right)$ were extracted and all are shown in Figure 3g-i. ${ }^{[3]}$ As shown in Figure 3g, 2D CdTe photodetector shows a photoswitching ratio of 27 and 5300 at room temperature and $80 \mathrm{~K}\left(P=132.3 \mathrm{~mW} \mathrm{~cm}{ }^{-2}\right)$, respectively. Besides, we studied the relationship between photoswitching ratio and voltage bias at $80 \mathrm{~K}$. As shown in Figure S5 (Supporting Information), a large photoswitching ratio around $10^{5}$ is obtained when the voltage bias is brought to $10 \mathrm{~V}$. Furthermore, responsivity $(R)$ is given by the formula: $R=I_{\mathrm{ph}} /(P S)$, where $S$ is the 
a
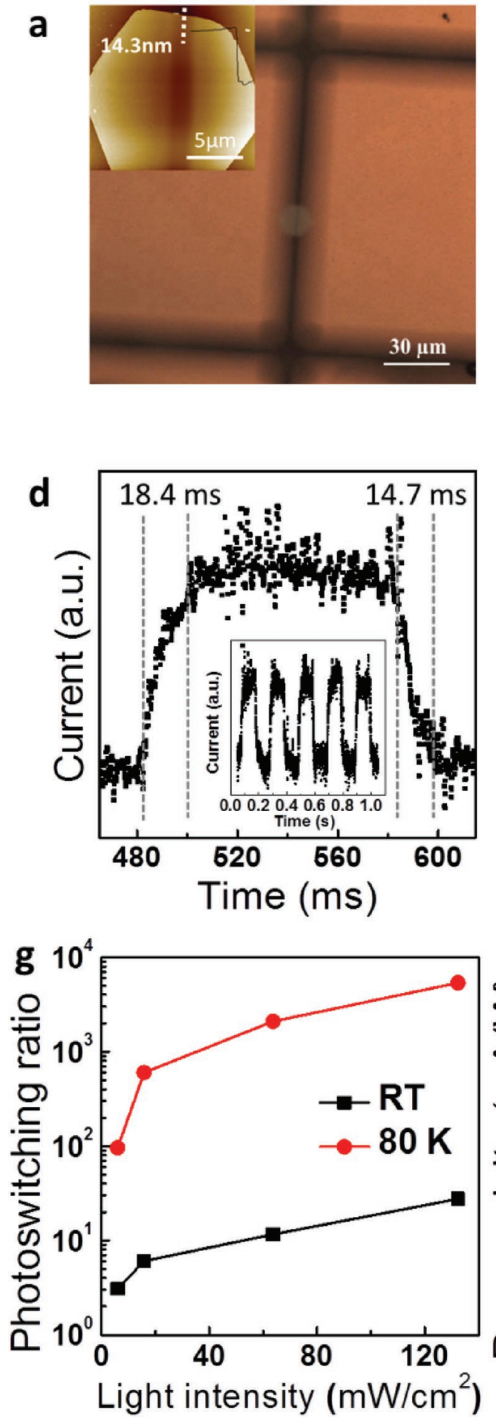
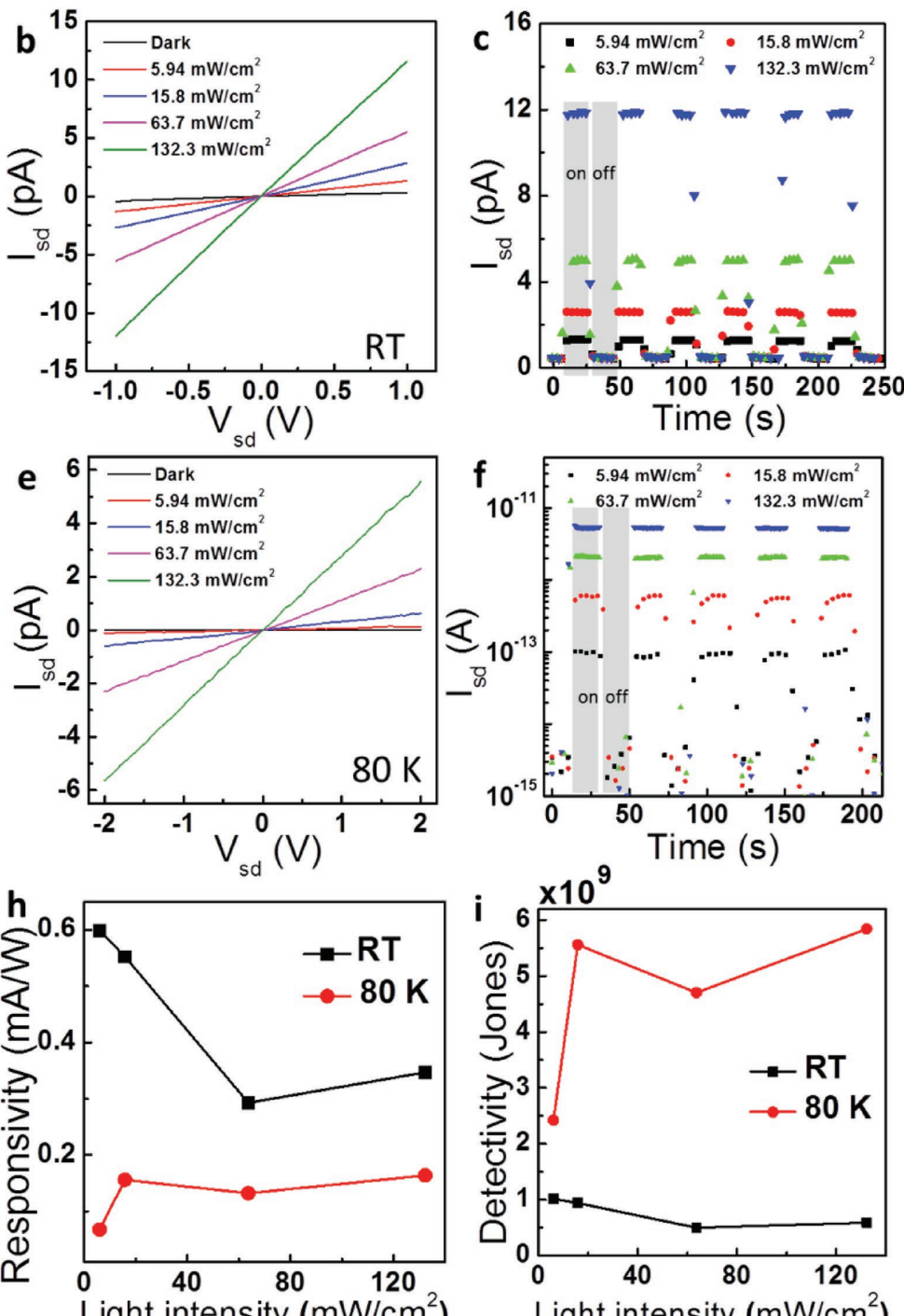

Light intensity $\left(\mathrm{mW} / \mathrm{cm}^{2}\right)$
Light intensity $\left(\mathrm{mW} / \mathrm{cm}^{2}\right)$

Figure 3. Photoresponse of 2D CdTe nanosheets photodetector with $473 \mathrm{~nm}$ laser. a) OM image of 2D CdTe photodetector with corresponding AFM image and height profiles as the inset. b) $I_{s d}-V_{s d}$ curves of CdTe photodetector in the dark and in the presence of laser with different intensities at room temperature. c) Time-dependent photoresponse of CdTe photodetector with a voltage bias of $1 \mathrm{~V}$ at room temperature. "On" and "off" correspond to laser on and off. d) A separated response and reset cycle: $\tau_{\text {rising }}=18.4 \mathrm{~ms}, \tau_{\text {decay }}=14.7 \mathrm{~ms}$, which corresponds to $100 \%$ rising and decay. Inset is its corresponding five cycles. e) $I_{\text {sd }}-V_{\text {sd }}$ Curves of $\mathrm{CdTe}$ photodetector in the dark and in the presence of laser with different intensities at $80 \mathrm{~K}$. f) Time-dependent photoresponse of CdTe photodetector with a voltage bias of $2 \mathrm{~V}$ at $80 \mathrm{~K}$. The plots of g) photoswitching ratio, h) responsivity, and i) detectivity versus laser power densities at room temperature and $80 \mathrm{~K}$, respectively.

active area, $I_{\mathrm{ph}}$ and $P$ are as defined above. In view of optically active area of photodetector $\left(7 \times 10^{-8} \mathrm{~cm}^{2}\right)$, the maximal responsivity at room temperature and $80 \mathrm{~K}^{2}$ are $0.6 \mathrm{~mA} \mathrm{~W}^{-1}$ $\left(P=5.94 \mathrm{~mW} \mathrm{~cm}^{-2}\right)$ and $0.16 \mathrm{~mA} \mathrm{~W}^{-1}\left(P=132.3 \mathrm{~mW} \mathrm{~cm}^{-2}\right)$, respectively. This low responsivity can be attributed to the low absorption cross section of 2D ultrathin structure. As an important figure of merit in evaluating the performance of photodetectors, specific detectivity is defined as: $D^{*}=(S \Delta f)^{1 / 2} / \mathrm{NEP}$. Here, $\Delta f$ and NEP are the electrical bandwidth (in $\mathrm{Hz}$ ) and the noise equivalent power, respectively. By assuming that the dark current contributes mainly to the shot noise, ${ }^{[44]}$ specific detectivity can be expressed as $D^{*}=I_{\mathrm{ph}} S^{1 / 2} / P S\left(2 q I_{\text {dark }}\right)^{1 / 2}$. In our case, the maximal $D *$ values are $1.02 \times 10^{9}$ and $5.84 \times 10^{9}$ Jones at room temperature and $80 \mathrm{~K}$, respectively. By comparing with previous 2D nonlayered materials, as shown in Table 1, 2D CdTe nanosheets show larger lateral size and thinner thickness, and the corresponding photodetectors display ultralow dark current, relatively high detectivity, and fast photoresponse speed. The above discussions demonstrate the prominent optoelectronic performance of 2D CdTe nanosheets.

In view of its $2 \mathrm{D}$ planar geometry, the compatibility of CdTe nanosheets with flexible substrates and traditional microfabrication techniques was also studied. Here, the excellent flexibility and insulating nature of mica sheets make it an ideal platform for flexible device. Another in situ fabricated 2D CdTe photodetector was fabricated and shown in Figure 4a. The device was characterized before and after bending with $V_{\mathrm{b}}=1 \mathrm{~V}$ and $P=132.3 \mathrm{~mW} \mathrm{~cm}{ }^{-2}$. The corresponding time-dependent 
Table 1. Comparison of 2D CdTe photodetectors with other works.

\begin{tabular}{|c|c|c|c|c|}
\hline Materials & Dimensions & Measurement condition & Photodetection performance & Ref. \\
\hline \multirow[t]{2}{*}{ CdTe } & $L: 5-11 \mu \mathrm{m}, T: 5-15 \mathrm{~nm}$ & $473 \mathrm{~nm}, V_{\mathrm{sd}}: 1 \mathrm{~V}$ & $I_{\text {dark }}: \approx 10^{-13} \mathrm{~A}, I_{\text {laser }} / I_{\text {dark }}: 27, R: 0.6 \mathrm{~mA} \mathrm{~W}^{-1}, D *: 10^{9}$ Jones, $\tau_{\text {rising }}: 18.4 \mathrm{~ms}, \tau_{\text {decay }}: 14.7 \mathrm{~ms}$ & This work \\
\hline & & $473 \mathrm{~nm}, V_{\mathrm{sd}}: 2 \mathrm{~V}, 80 \mathrm{~K}$ & $I_{\text {dark }}:<10^{-14} \mathrm{~A}, I_{\text {laser }} / I_{\text {dark }}: 5300, R: 0.16 \mathrm{~mA} \mathrm{~W}-1, D *: 5.8 \times 10^{9}$ Jones & This work \\
\hline CdSe & $L: \approx 4 \mu \mathrm{m}, T: \approx 70 \mathrm{~nm}$ & $450 \mathrm{~nm}, V_{\mathrm{sd}}=5 \mathrm{~V}$ & $I_{\text {dark }}: \approx 10^{-7} \mathrm{~A}, I_{\text {laser }} / I_{\text {dark }}: 1.7, R: 500 \mathrm{~A} \mathrm{~W}^{-1}, \tau_{\text {rising }}=\tau_{\text {decay }}: 24 \mathrm{~ms}$ & [33] \\
\hline $\mathrm{Te}$ & $L: 6-10 \mu \mathrm{m}, T: 30-80 \mathrm{~nm}$ & $473 \mathrm{~nm}, V_{\mathrm{sd}}=2 \mathrm{~V}$ & $I_{\text {dark: }}: 10^{-6} \mathrm{~A}, I_{\text {laser }} / I_{\text {dark }}: 1.6, R: 160 \mathrm{~A} \mathrm{~W}^{-1}, \tau_{\text {rising }}: 4.4 \mathrm{~s}, \tau_{\text {decay }}: 2.8 \mathrm{~s}$ & [31] \\
\hline PbS & $L: \approx 2-2.6 \mu \mathrm{m}, T: 10-35 \mathrm{~nm}$ & $800 \mathrm{~nm}, V_{\mathrm{sd}}=0.1 \mathrm{~V}$ & $I_{\text {dark: }}: \approx 10^{-9} \mathrm{~A}, I_{\text {laser }} / I_{\text {dark }}:<2, R: 1600 \mathrm{~A} \mathrm{~W}^{-1}, D^{*}: 10^{11}$ Jones, $\tau_{\text {rising }}=\tau_{\text {decay }}: \approx 300 \mathrm{~ms}$ & [34] \\
\hline
\end{tabular}

Note: $L$ : lateral dimension, $T$ : thickness, $I_{\text {dark }}$ dark current, $D^{*}$ : specific detectivity, $R$ : responsivity.

photoresponse is displayed in Figure 4b. After being bent 50 times, $I_{\text {laser }}$ and $I_{\text {dark }}$ both decreased slightly, which can be attributed to the destructive contacts between nanosheet and electrodes. In spite of this slight decrease, the device still exhibits a high sensitivity and durability, showing great potential in the application of flexible and wearable optoelectronic devices. Besides, the field effect of CdTe nanosheets was also examined. The as-grown CdTe nanosheets were transferred onto a silicon substrate from mica sheet, followed by the fabrication of drain and source electrodes using standard electron-beam lithography (EBL) and metal deposition of $\mathrm{Cr} / \mathrm{Au}$
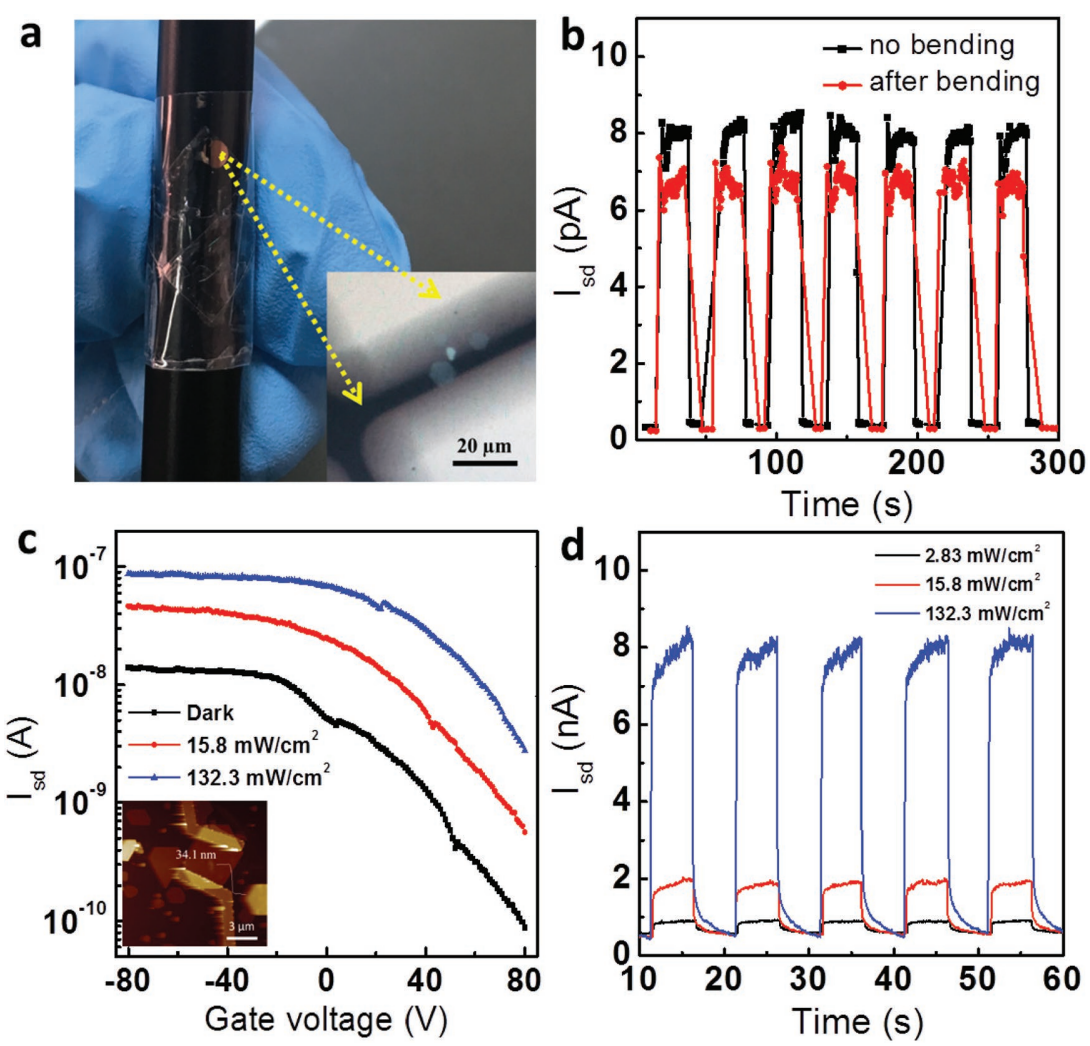

Figure 4. The compatibility measurement of $\mathrm{CdTe}$ nanosheets with $\mathrm{a}, \mathrm{b})$ flexible substrates and c,d) traditional microfabrication techniques. a) Photograph of 2D CdTe photodetector. Inset: corresponding $\mathrm{OM}$ image of a single 2D CdTe photodetector. b) Time-dependent photoresponse of CdTe photodetector with voltage bias of $1 \mathrm{~V}$ before and after being bent 50 times. $P=132.3 \mathrm{~mW} \mathrm{~cm}^{-2}$. c) Transfer characteristic curve of 2D CdTe photodetector in the dark and in the presence of $473 \mathrm{~nm}$ laser with different intensities, which exhibits an obvious p-type conducting behavior. Voltage bias is $5 \mathrm{~V}$. Inset: AFM image and height profiles of CdTe nanosheet. d) Timedependent photoresponse of CdTe photodetector under zero gate voltage. Voltage bias is $1 \mathrm{~V}$.
(8 nm/50 nm). The heavily doped silicon substrate with $300 \mathrm{~nm}$ thick $\mathrm{SiO}_{2}$ is applied as a gate electrode. Further details can be found in the Experimental Section. The AFM image of our developed device with a channel thickness around $34 \mathrm{~nm}$ is presented as the inset of Figure 4c. The $I_{\mathrm{sd}}-V_{\mathrm{sd}}$ curves of the device were measured under different laser intensities, still exhibits very sensitive optoelectronic properties. Figure $4 \mathrm{c}$ depicts the transfer characteristic curve $\left(I_{\mathrm{sd}}-V_{\text {gate }}\right)$ of the photodetector at an operation voltage bias of $5 \mathrm{~V}$, which exhibits a typical p-type conducting behavior with an on/ off ratio over two orders. Figure $4 \mathrm{~d}$ exhibits its time-dependent photoresponse under $V_{\text {sd }}=1 \mathrm{~V}$ and $V_{\text {gate }}=0 \mathrm{~V}$, showing a highly stable and reversible photoswitching. A separated response and reset cycle are given in Figure S6b (Supporting Information) with a response time of $0.1 \mathrm{~s}$ and a longer recovery time, which can be attributed to the introduction of trap states during the transfer progress. Moreover, its low-temperature performance was also analyzed. As displayed in Figure S6c,d (Supporting Information), 2D CdTe photodetector shows a stable photoswitching with a ratio around $10^{5}$ under a high voltage bias. Given a laser intensity of $132.3 \mathrm{~mW} \mathrm{~cm} \mathrm{~cm}^{-2}$, the corresponding responsivity and specific detectivity are $94 \mathrm{~mA} \mathrm{~W}^{-1}, 4.3 \times 10^{11}$ Jones for $V_{\text {sd }}=10 \mathrm{~V}$, and $429 \mathrm{~mA} \mathrm{~W}^{-1}, 7.1 \times 10^{11}$ Jones for $V_{\mathrm{sd}}=20 \mathrm{~V}$, respectively. Here, the active area of the device used in Figure $4 \mathrm{c}$ is $1.2 \times 10^{-7} \mathrm{~cm}^{2}$. The above discussions demonstrate its high compatibility with traditional microfabrication techniques, laying the foundation for high-performance photovoltaic devices by combining with other ultrathin 2DLMs.

In summary, we successfully synthesized ultrathin nonlayered CdTe nanosheets via an effective van der Waals epitaxy method. Single-crystalline nanosheets with uniform thickness as well as large lateral dimensions are obtained, providing not only an attractive structural block for a new generation of electronics devices, but also a promising platform 
for both fundamental research and technical applications due to strong dimensional confinement and ultrahigh specific surface area. Moreover, the fabricated ultrathin CdTe photodetector shows not only ultralow dark current $(\approx 100 \mathrm{fA})$, high detectivity, stable photoswitching, and ultrafast photoresponse speed $\left(\tau_{\text {rising }}=18.4 \mathrm{~ms}, \tau_{\text {decay }}=14.7 \mathrm{~ms}\right)$, but also high compatibility with flexible substrates and traditional microfabrication techniques, indicating its significant potential in the applications of optoelectronic devices.

\section{Experimental Section}

Synthesis of 2D CdTe Nanosheets: CdTe Nanosheets were obtained by vapor transport method in a horizontal vacuum quartz tube furnace with two heating zones. Weighed amount of Te powder $(99.99 \%$, Alfa Aesar) and CdTe powder (99.99\%, Alfa Aesar) were mixed evenly in a quartz boat before loaded in the center of first heating zone. Layered fluorophlogopite mica $\left[\mathrm{KMg}_{3}\left(\mathrm{AlSi}_{3} \mathrm{O}_{10}\right) \mathrm{F}_{2}\right]$ sheets were positioned 6-9 cm downstream away from the second heating zone. The tube was sealed, evacuated, and flushed with high-purity Ar gas in order to provide oxygen-free environment. In the growth process, Ar gas was used as the carrier gas with a constant flow rate of $20-50 \mathrm{sccm}$. The whole reaction process was carried out under the furnace temperature of $690-720^{\circ} \mathrm{C}$ for the first heating center and $560-590^{\circ} \mathrm{C}$ for the second heating center. The reaction continued for $15 \mathrm{~min}$. And then the furnace naturally cooled down to room temperature. Eventually, 2D CdTe nanosheets were deposited on the mica substrates.

Characterizations: The morphology, composition, and crystal microstructure of 2D CdTe nanosheets were characterized by $\mathrm{OM}$ (Olympus BX51M), AFM (Dimension 3100), XPS (ESCALAB $250 \mathrm{Xi}$ ), Raman (Renishaw InVia, $532 \mathrm{~nm}$ excitation laser), photoluminescence (Renishaw InVia plus, $514 \mathrm{~nm}$ excitation laser), high-resolution transmission electron microscope (JEM-2100F), SAED, and electron EDX attached to the TEM.

Transfer Process of CdTe Nanosheets: For the TEM measurements, 2\% $\mathrm{HF}$ solution was used to disperse and transfer $\mathrm{CdTe}$ nanosheets from the mica substrates onto copper grid. For the field-effect measurements, poly(methyl methacrylate) (PMMA) was spin-coated on $\mathrm{CdTe} /$ mica surface at first. Then, $2 \% \mathrm{HF}$ solution was used to etch the mica substrate until the PMMA-capped CdTe film completely leaves mica sheet, followed by supporting the film with a $300 \mathrm{~nm} \mathrm{SiO} / \mathrm{Si}$ substrate. And then, this substrate with CdTe film on the surface was dried on a hot-plate $\left(100{ }^{\circ} \mathrm{C}\right.$ for $\left.10 \mathrm{~min}\right)$. Finally, hot acetone was used to remove the PMMA $\left(80^{\circ} \mathrm{C}\right.$ for $\left.10 \mathrm{~min}\right)$.

Device Fabrication and Measurements: The drain and source electrodes of devices were defined by copper grid shadow mask and standard EBL, respectively. And subsequently, $\mathrm{Cr} / \mathrm{Au}(8 \mathrm{~nm} / 50 \mathrm{~nm})$ was deposited by thermal evaporation. The electrical properties were measured in a probe station (Lakeshore, TTP4) with a sealed chamber. The parameters were analyzed using a Keithley 4200 semiconductor analyzer. A $473 \mathrm{~nm}$ laser with a tunable laser attenuator was employed to investigate its photoresponse. Here, the efficient power was obtained by taking account of optical active area. All the measurements were carried out under high vacuum $\left(\approx 10^{-6}\right.$ Torr $)$ to eliminate the possible influence of air.

\section{Supporting Information}

Supporting Information is available from the Wiley Online Library or from the author.

\section{Acknowledgements}

R.C. and Y.W. contributed equally to this work. This work was supported by National Natural Science Foundation of China (Nos. 61625401,
61474033, and 61574050), Ministry of Science and Technology of China (No. 2016YFA0200700), Strategic Priority Research Program of the Chinese Academy of Sciences (Grant No. XDA09040201), and CAS Key Laboratory of Nanosystem and Hierarchical Fabrication. The authors also gratefully acknowledge the support of Youth Innovation Promotion Association CAS.

\section{Conflict of Interest}

The authors declare no conflict of interest.

\section{Keywords}

2D nanostructures, cadmium telluride, nonlayered materials, photodetectors, van der Waals epitaxy

Received: June 5, 2017

Revised: June 19, 2017

Published online:

[1] K. S. Novoselov, A. K. Geim, S. V. Morozov, D. Jiang, Y. Zhang, S. V. Dubonos, I. V. Grigorieva, A. A. Firsov, Science 2004, 306, 666.

[2] T. Ohta, A. Bostwick, T. Seyller, K. Horn, E. Rotenberg, Science 2006, $313,951$.

[3] K. F. Mak, C. Lee, J. Hone, J. Shan, T. F. Heinz, Phys. Rev. Lett. 2010 105,136805

[4] G. Eda, H. Yamaguchi, D. Voiry, T. Fujita, M. Chen, M. Chhowalla, Nano Lett. 2011, 11, 5111.

[5] H. R. Gutierrez, N. Perea-Lopez, A. L. Elias, A. Berkdemir, B. Wang, R. Lv, F. Lopez-Urias, V. H. Crespi, H. Terrones, M. Terrones, Nano Lett. 2013, 13, 3447.

[6] L. Li, Y. Yu, G. J. Ye, Q. Ge, X. Ou, H. Wu, D. Feng, X. H. Chen, Y. Zhang, Nat. Nanotechnol. 2014, 9, 372.

[7] B. Radisavljevic, A. Radenovic, J. Brivio, I. V. Giacometti, A. Kis, Nat. Nanotechnol. 2011, 6, 147.

[8] M. S. Choi, G. H. Lee, Y. J. Yu, D. Y. Lee, S. H. Lee, P. Kim, J. Hone, W. J. Yoo, Nat. Commun. 2013, 4, 1624

[9] R. Cheng, F. Wang, L. Yin, K. Xu, T. A. Shifa, Y. Wen, X. Zhan, J. Li, C. Jiang, Z. Wang, J. He, Appl. Phys. Lett. 2017, 110, 173507.

[10] F. K. Perkins, A. L. Friedman, E. Cobas, P. M. Campbell, G. G. Jernigan, B. T. Jonker, Nano Lett. 2013, 13, 668.

[11] O. Lopez-Sanchez, D. Lembke, M. Kayci, A. Radenovic, A. Kis, Nat. Nanotechnol. 2013, 8, 497.

[12] M. Massicotte, P. Schmidt, F. Vialla, K. G. Schädler, A. Reserbat-Plantey, K. Watanabe, T. Taniguchi, K.-). Tielrooij, F. H. Koppens, Nat. Nanotechnol. 2015, 11, 42

[13] C.-H. Lee, G.-H. Lee, A. M. Van Der Zande, W. Chen, Y. Li, M. Han X. Cui, G. Arefe, C. Nuckolls, T. F. Heinz, J. Guo, J. Hone, P. Kim, Nat. Nanotechnol. 2014, 9, 676.

[14] Y. Liu, N. O. Weiss, X. Duan, H.-C. Cheng, Y. Huang, X. Duan, Nat Rev. Mater. 2016, 1, 16042

[15] Y. Kim, S. S. Cruz, K. Lee, B. O. Alawode, C. Choi, Y. Song, J. M. Johnson, C. Heidelberger, W. Kong, S. Choi, K. Qiao, I. Almansouri, E. A. Fitzgerald, J. Kong, A. M. Kolpak, J. Hwang, J. Kim, Nature 2017, 544, 340

[16] L. Li, G. J. Ye, V. Tran, R. Fei, G. Chen, H. Wang, J. Wang, K. Watanabe, T. Taniguchi, L. Yang, X. H. Chen, Y. Zhang, Nat. Nanotechnol. 2015, 10, 608.

[17] L. Li, F. Yang, G. J. Ye, Z. Zhang, Z. Zhu, W. Lou, X. Zhou, L. Li, K. Watanabe, T. Taniguchi, K. Chang, Y. Wang, X. H. Chen, Y. Zhang, Nat. Nanotechnol. 2016, 11, 593. 
[18] Y. Zhao, M. Boccard, S. Liu, J. Becker, X.-H. Zhao, C. M. Campbell, E. Suarez, M. B. Lassise, Z. Holman, Y.-H. Zhang, Nat. Energy 2016, 1, 16067

[19] B. Zhang, P. Lu, H. Liu, L. Jiao, Z. Ye, M. Jaime, F. F. Balakirev, H. Yuan, H. Wu, W. Pan, Y. Zhang, Nano Lett. 2015, 15, 4381.

[20] Y.-D. Zheng, Y. H. Chang, B. D. McCombe, R. F. C. Farrow, T. Temofonte, F. A. Shirlland, Appl. Phys. Lett. 1986, 49, 1187.

[21] Q.-F. Sun, Y.-X. Li, W. Long, J. Wang, Phys. Rev. B 2011, 83, 115315.

[22] C. Tan, H. Zhang, Nat. Commun. 2015, 6, 7873.

[23] F. Wang, J. H. Seo, G. Luo, M. B. Starr, Z. Li, D. Geng, X. Yin, S. Wang, D. G. Fraser, D. Morgan, Z. Ma, X. Wang, Nat. Commun. 2016, 7, 10444

[24] Z. Y. Al Balushi, K. Wang, R. K. Ghosh, R. A. Vilá, S. M. Eichfeld, J. D. Caldwell, X. Qin, Y.-C. Lin, P. A. DeSario, G. Stone, Nat. Mater. 2016, 15, 1166.

[25] J. Kim, C. Bayram, H. Park, C. W. Cheng, C. Dimitrakopoulos, J. A. Ott, K. B. Reuter, S. W. Bedell, D. K. Sadana, Nat. Commun. 2014, 5, 4836.

[26] M. I. Utama, Q. Zhang, J. Zhang, Y. Yuan, F. J. Belarre, J. Arbiol, Q. Xiong, Nanoscale 2013, 5, 3570.

[27] Y. Zhou, Y. Nie, Y. Liu, K. Yan, J. Hong, C. Jin, Y. Zhou, J. Yin, Z. Liu, H. Peng, ACS Nano 2014, 8, 1485.

[28] Y. Shi, W. Zhou, A. Y. Lu, W. Fang, Y. H. Lee, A. L. Hsu, S. M. Kim, K. K. Kim, H. Y. Yang, L. J. Li, J. C. Idrobo, J. Kong, Nano Lett. 2012, 12, 2784.

[29] M. Lin, D. Wu, Y. Zhou, W. Huang, W. Jiang, W. Zheng, S. Zhao, C. Jin, Y. Guo, H. Peng, Z. Liu, J. Am. Chem. Soc. 2013, 135, 13274.

[30] J. Wu, H. Yuan, M. Meng, C. Chen, Y. Sun, Z. Chen, W. Dang, C. Tan, Y. Liu, J. Yin, Y. Zhou, S. Huang, H. Q. Xu, Y. Cui,
H. Y. Hwang, Z. Liu, Y. Chen, B. Yan, H. Peng, Nat. Nanotechnol. 2017, 12, 530 .

[31] Q. Wang, M. Safdar, K. Xu, M. Mirza, Z. Wang, J. He, ACS Nano 2014, 8, 7497.

[32] Q. Wang, K. Xu, Z. Wang, F. Wang, Y. Huang, M. Safdar, X. Zhan, F. Wang, Z. Cheng, J. He, Nano Lett. 2015, 15, 1183.

[33] D. D. Zhu, J. Xia, L. Wang, X. Z. Li, L. F. Tian, X. M. Meng, Nanoscale 2016, 8, 11375.

[34] Y. Wen, Q. Wang, L. Yin, Q. Liu, F. Wang, F. Wang, Z. Wang, K. Liu, K. Xu, Y. Huang, T. A. Shifa, C. Jiang, J. Xiong, J. He, Adv. Mater. 2016, 28, 8051.

[35] Z. Bai, D. Wang, Phys. Status Solidi A 2012, 209, 1982.

[36] J. G. Werthen, J.-P. Haring, A. L. Fahrenbruch, R. H. Bube, J. Phys. D 1983, 16, 2391.

[37] K. Varazo, M. D. Lay, T. A. Sorenson, J. L. Stickney, J. Electroanal. Chem. 2002, 522, 104

[38] M. Setty, A. Sinha, Thin Solid Films 1986, 144, 7.

[39] E. Campos-González, F. de Moure-Flores, L. E. Ramírez-Velázquez, K. Casallas-Moreno, A. Guillén-Cervantes, J. Santoyo-Salazar, G. Contreras-Puente, O. Zelaya-Angel, Mater. Sci. Semicond. Process. 2015, 35, 144

[40] M. Levy, N. Amir, E. Khanin, A. Muranevich, Y. Nemirovsky, R. Beserman, J. Cryst. Growth 1998, 187, 367.

[41] C. Frausto-Reyes, J. R. Molina-Contreras, C. Medina-Gutierrez, S. Calixto, Spectrochim. Acta, Part A 2006, 65, 51.

[42] W. Zheng, Z. Zhang, R. Lin, K. Xu, J. He, F. Huang, Adv. Electron. Mater. 2016, 2, 1600291.

[43] G. Konstantatos, E. H. Sargent, Nat. Nanotechnol. 2010, 5, 391.

[44] F. Wang, Z. Wang, K. Xu, F. Wang, Q. Wang, Y. Huang, L. Yin, J. He, Nano Lett. 2015, 15, 7558. 